\title{
RNF125 is a Ubiquitin-Protein Ligase that Promotes p53 Degradation
}

\author{
Liuzhong Yang ${ }^{\mathrm{a}}$ Bing Zhou ${ }^{\mathrm{b}}$ Xiaorui $\mathrm{Li}^{\mathrm{a}}$ Zhihong Lua Weiwei $\mathrm{Li}^{\mathrm{a}}$ Xiaoqing Huo \\ Zhanhui Miao ${ }^{a}$ \\ aCancer Department of First Affiliated Hospital of Xinxiang Medical College, Xinxiang, bepartment of \\ General Surgery, Xinxiang Medical University, Xinxiang, Henna, China
}

\section{Key Words}

RNF125 • p53 • E3 ubiquitin ligase • Proteasome degradation

\begin{abstract}
Background/Aims: Although early studies show that $\mathrm{Mdm} 2$ is the primary E3 ubiquitin ligase for the p53 tumor suppressor, an increasing amount of data suggests that p53 ubiquitination and degradation are more complex than once thought. Here, we investigated the role of RNF125, a non-Mdm2 ubiquitin-protein ligase, in the regulation of p53. Methods and Results: RNF125 physically interacted with p53 in exogenous/endogenous co-immunoprecipitation (IP) and GST-pull down assay, and a C72/75A mutation of RNF125 did not interfere with this interaction. Expression of RNF125 decreased the level of p53 in a dose-dependent manner, whereas knockdown of RNF125 by RNA interference increased the level of p53. As shown by Western blotting and ubiquitin assay, RNF125 ubiquitinated p53 and targeted it for proteasome degradation. Furthermore, RNF125 repressed p53 functions including p53dependent transactivation and growth inhibition. Conclusion: Our data suggest that RNF125 negatively regulates p53 function through physical interaction and ubiquitin-mediated proteasome degradation.
\end{abstract}

Copyright $\odot 2015$ S. Karger AG, Basel

\section{Introduction}

The p53 is a key regulator of cell cycle control, apoptosis and genomic stability in response to various cellular stresses [1]. Tight regulation of p53 is essential for maintaining normal cell growth and this occurs primarily through post-translational modifications of p53, including ubiquitination, acetylation, phosphorylation, sumoylation, neddylation, methylation and glycosylation [2]. It is well known that the ubiquitin-proteasome pathway

Dr. Zhanhui Miao

KARGER 125
Cancer Department of First Affiliated Hospital of Xinxiang Medical College, Xinxiang, Henna, 453100 (China)

E-Mail zhanhuimiao@hotmail.com 
plays a major part in regulating p53 [3]. Three independent studies identified the mouse double minute protein $2(\mathrm{Mdm} 2)$ as the principal endogenous E3 ligase p53, for which Mdm2 shows a high degree of specificity [4-6]. Mdm2 is a transcriptional target of p53; thus, p53 activity controls the expression and protein level of its own negative regulator, providing an elegant feedback loop [7]. Mdm2 inhibits the G1 arrest and apoptosis functions of the p53 [8]. The Mdm2-p53 complex also inhibits p53-mediated transactivation [9].

Recent data suggest that Mdm2-mediated ubiquitination is not the only important factor for $\mathrm{p} 53$ regulation $[10,11]$. In addition to $\mathrm{Mdm} 2$, other $\mathrm{E} 3$ ligases have been shown to exhibit specificity for p53 and promote its proteasome-mediated degradation. Indeed, the recently discovered E3 ligases COP1, Pirh2, Arf-BP1, CARPs have clearly been shown to contribute to the efficient control of p53 levels in tissue culture and in vitro biochemical experiments [12-15]. However, it is sitll uncertain whether there are other E3 ligases that regulate p53. To screen new E3 ligases that can regulate the activity of p53 in Mdm2-independent manner, a spleen cDNA library was screened using yeast two hybrid system with a p53 plasmid as bait (data not shown). RNF125 was isolated and its interaction with p53 was detected. In the present studies, we further demonstrate that RNF125 physically interacts with p53 and downregulates its expression by promoting its degradation through proteasome-ubiquitin system, providing a potential role for RNF125 in promoting tumorigenesis.

In the present studies, we further demonstrate that RNF125 physically interacts with p53 and downregulates its expression by promoting its degradation through proteasomeubiquitin system, providing a novel mechanism for RNF125 in promoting tumorigenesis.

\section{Materials and Methods}

\section{Antibodies and reagents}

Antibodies to Flag and HA were purchased from Sigma (St. Louis, MO). Anti-Myc, anti-ubiquitin, anti- $\beta$ tubulin antibodies were obtained from Santa Cruz Biotechnology (Santa Cruz, CA). Anti-GST antibodies, peroxidase-conjugated goat anti-mouse and goat anti-rabbit immunoglobulin were acquired from CWBIO (CWBIO, China). p53 and RNF125 was obtained from Santa cruz (Santa Cruz, CA).

Lipofectamine 2000 transfection reagent, RNase A, RPMI 1640 medium, DMEM culture medium, and fetal bovine serum were from Invitrogen. MG132 was purchased from Promega. Etoposide (Etop) and adriamycin (ADR) were obtained from Sigma. Fetal bovine serum was purchased from Hyclone. Protease inhibitor cocktail was acquired from Roche. The dual luciferase reporter assay kit was obtained from Promega. The GenBank accession number for mRNF125 is AB259692.

\section{Cell culture and transfection}

The 293 human embryonic kidney cell line and HCT116 colon carcinoma cell line were grown in DMEM or McCoy's 5A supplemented with $10 \%$ fetal bovine serum and maintained at $37{ }^{\circ} \mathrm{C}$ under $5 \% \mathrm{CO}_{2}$ in a humidified incubator. For transfections, cells were plated to $85-90 \%$ confluence and transfected with Lipofectamine 2000. For transient knockdown of RNF125, RNF125 siRNA oligonucleotide was transfected following the manufacture's instructions.

\section{IP and immunoblot analysis}

Cell extracts were prepared in NP-40 lysis buffer containing $50 \mathrm{mM}$ Tris- $\mathrm{HCl}(\mathrm{pH}$ 8.0), $150 \mathrm{mM} \mathrm{NaCl}$, and $1 \%$ NP-40 in the presence of Complete ${ }^{\circledR}$ protease inhibitor cocktail. Supernatants of cell lysates were subjected to IP with anti-Flag. For endogenous IP, anti-p53 antibody and protein A/G Sepharose beads were added to the lysates instead. The immunoprecipitated proteins were blotted onto PVDF membranes after being separated by $8-12 \%$ SDS-PAGE, and were then detected with anti-Flag, anti-Myc, anti-HA, or anti- $\beta$ tubulin antibody.

\section{GST-pull down}

For GST-pull down experiments, cell lysates containing Flag-p53 were incubated for $2 \mathrm{~h}$ at $4{ }^{\circ} \mathrm{C}$ with 1 $\mu \mathrm{g}$ purified GST or GST-RNF125 fusion proteins bound to glutathione beads. The immunoprecipitates were washed with NP-40 lysis buffer and then subjected to immunoblotting analysis. 
In vivo ubiquitination assays

Cells were co-transfected with Flag-p53, HA-RNF125, and Myc-ubiquitin. After 20 h, cells were grown in medium containing MG132 $(20 \mu \mathrm{M})$ for $12 \mathrm{~h}$. Immunoprecipitates with anti-Flag agarose were analyzed via immunoblotting with anti-Flag and Myc antibodies. Flag-p53 was used as a loading control.

\section{Luciferase reporter assays}

HCT116 cells were plated onto 24-well plates and transfected with p53-luciferase reporter plasmids (100 ng p53-luc and 2 ng pRL-TK) together with 100 ng plasmid encoding HA-RNF125. After $24 \mathrm{~h}$, transfected cells were collected, and luciferase activity was measured using the dual luciferase reporter assay kit.

\section{Statistical Analysis}

The results were expressed as the mean \pm SE of at least three independent experiments. Statistical significance was inferred when $\mathrm{p}<0.05$.

\section{Results}

\section{RNF125 interacts with $p 53$ in vivo and in vitro}

To investigate whether RNF125 interacts with the p53 tumor suppressor protein, 293 cells were co-transfected with HA-tagged RNF125 and Flag-p53 or Flag-vector. Following transfection, co-IP was performed using anti-Flag agarose. As expected, RNF125 was detected in the IP complex with p53 but not with a negative control (Fig. 1a). To exclude the influence of tag, a reciprocal set of experiments was performed in which lysates of 293

Fig. 1. Association of RNF125 with p53. a, 293 cells were co-transfected with Flag-p53 or Flag-vector together with HA-RNF125. After $24 \mathrm{~h}$ of transfection, co-IP experiment was performed using anti-Flag agarose. The interaction was detected by immunoblotting with anti-Flag and anti-HA antibodies. b: Similar to (a) except that cells were transfected with Flag-RNF125 and Myc-p53 instead of Flag-p53 and HA-RNF125. c: Association of p53 with RNF125. d: 293 cells transfected with Flag-p53 expression vector were lysed by NP-40 lysis buffer. The GST or GST-RNF125 fusion protein absorbed from cell lysates were analyzed using immunoblotting with anti-FLAG antibody (top). Loading of GST proteins was assessed using immunoblotting with anti-GST antibody (bottom). e: Lysates from HCT116 cells were separated by SDS-PAGE and analyzed using immunoblotting with anti-p53 antibody. e: Analysis of the association of p53 with RNF125 mutant. Plasmids as indicated were transfected into 293 cells. Cell lysates were immunoprecipitated using an anti-Flag antibody and detected with the anti-HA antibody.

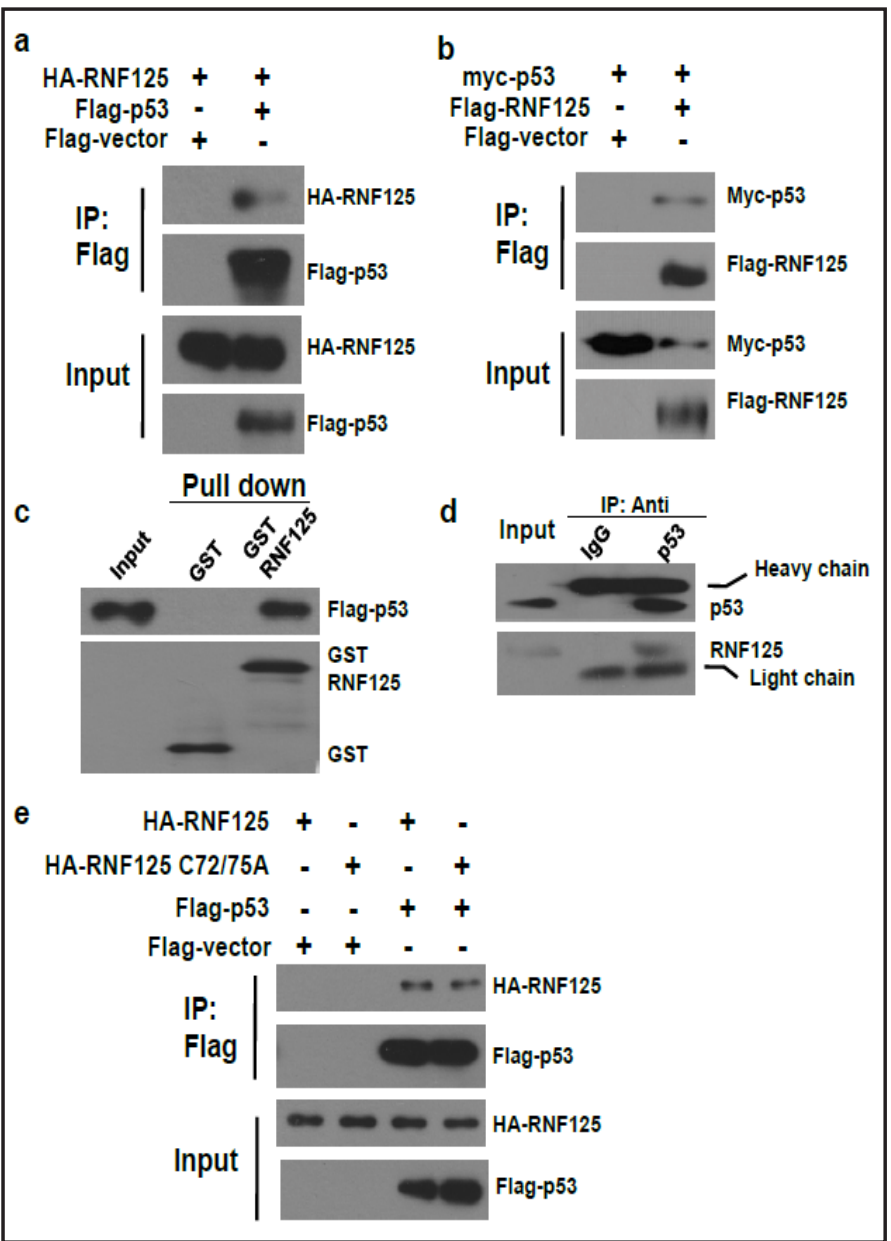


Fig. 2. RNF125 promotes p53 degradation. a: HCT116 cells were transfected with p53 and increasing amount of RNF125 expression plasmids $(0,0.5,1,1.5 \mu \mathrm{g})$. Cell lysates were separated by SDS-PAGE. The separated proteins were blotted onto membranes and then detected with anti-Flag and anti-HA antibodies. $\beta$-Tubulin was used as a loading control. b: Plasmids encoding Flalg-p53 and HA-RNF125 were co-transfected into HCT116 cells with WT or a point mutant of HA-RNF125 (C72/75A). Cells were harvested $36 \mathrm{~h}$ after transfection and proteins were analyzed using Western blot. $\beta$-Tubulin was used as a loading control. c: RNF125 expression in HCT116 cells treated with control siRNA,

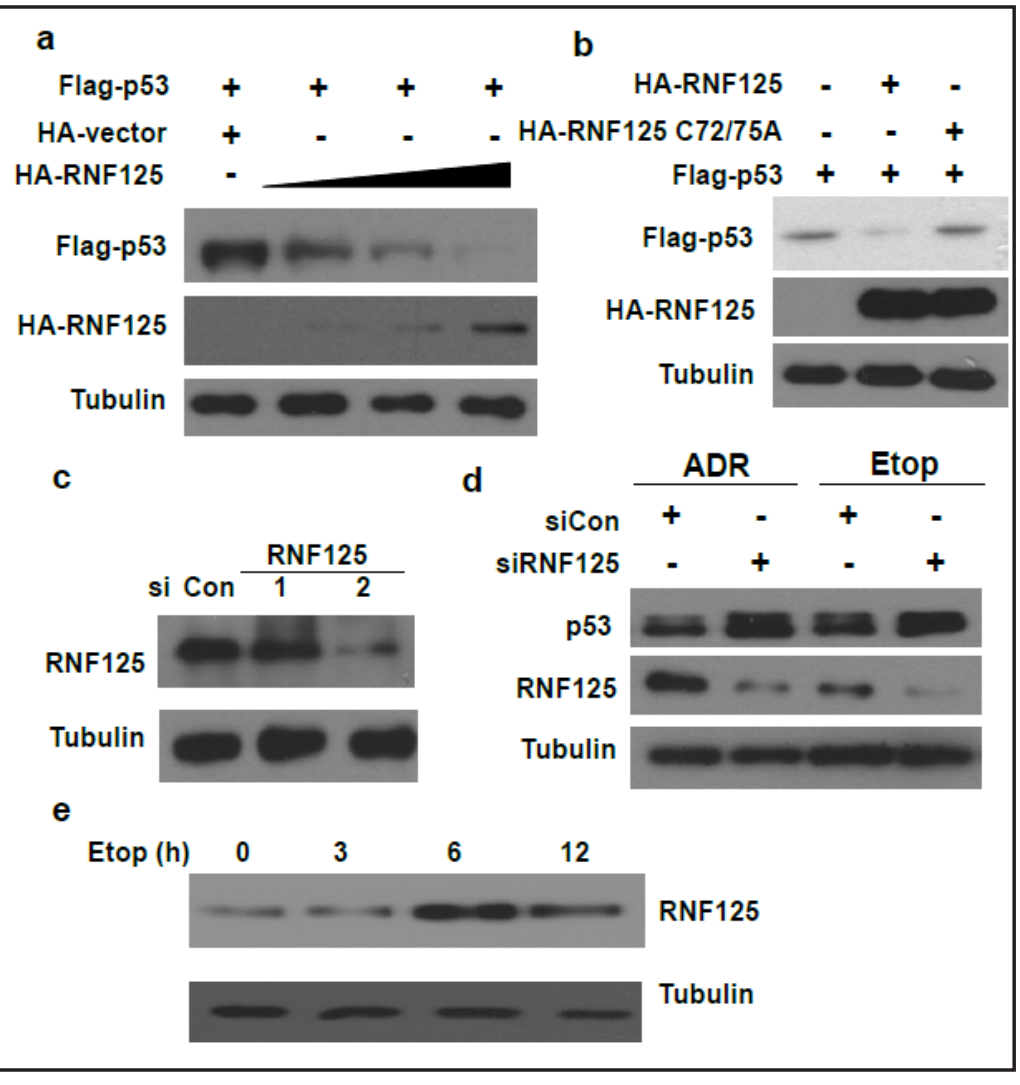
siRNA-1 or siRNAi-2. Cell lysates were separated by SDS-PAGE. The separated proteins were blotted onto the membranes and then detected with anti-HA antibodies. $\beta$-Tubulin was used as a loading control. $\mathrm{d}$ : HCT116 cells were transfected with siRNF125 or control siRNA. Seventy-two hours later, the drugs were added for an additional $6 \mathrm{~h}$. Whole cell lysates were analyzed using immunoblotting with p53, RNF125 and $\beta$-Tubulin antibody. e: RNF125 expression in HCT116 cells treated with Etop. HCT116 cells were treated with Etop and harvested at indicated time. Whole cell lysates were analyzed using immunoblotting with anti-RNF125 antibody. $\beta$-Tubulin was used as a loading control.

cells co-expressing Myc-p53 and Flag-RNF125 were immunoprecipitated with anti-Flag and examined with anti-Myc antibody. In Fig. 1b, p53 was also detected in the IP complex with RNF125 but not with a negative control. To evaluate whether RNF125 and p53 interact in vitro, cell lysates from 293 cells expressing Flag-p53 were incubated with GST or GST-RNF125 fusion protein. As shown in Fig. 1c, p53 protein bound to immobilized GST-RNF125 but not to GST alone. The results from the in vitro experiments further supported the association between RNF125 and p53. Then, the specificity of the interaction between endogenous p53 and RNF125 was also examined via co-IP analysis using p53-specific antibody and control normal IgG, which demonstrated that p53 was associated with endogenous RNF125 in HCT116 cells (Fig. 1d). These results showed that RNF125 physically interacts with p53.

\section{RNF125 downregulates expression of 553}

As a tumor suppressor, p53 plays a crucial role in preventing tumorigenesis by inducing apoptosis. We examined the effect of RNF125 on the expression of p53. Increasing amounts of RNF125 expression plasmids were co-transfected with the same amounts of p53 into HCT116 cells. A significant reduction of p53 was observed when it was co-transfected with RNF125 (Fig. 2a). To gain further insight into the mechanism of RNF125-mediated reduction of p53 protein levels in HCT116 cells, the effect of E3 ligase dead mutant was assayed. Previous studies have shown that RNF125 abrogates its ubiquitin-conjugating activity when Cys72 and Cys75 residues were substituted with alanine (C72/75A). Here, 
Fig. 3. RNF125 mediates ubiquintination of p53. a: RNF125 stimulates p53 degradation in a proteasome-dependent manner. Plasmids encoding a Flag-tagged version of p53 and either HA-RNF125 or HA-vector were co-transfected into HCT116 cells. After culturing with or without the proteasome inhibitor MG132, exogenous p53 and RNF125 levels were assessed by immunoblotting. b: Ubiquitin conjugation to p53 by RNF125. Plasmids encoding Flag-p53 and Myc-ubi were co-transfected into HCT116 cells with a plasmid encoding either HA-RNF125 or HA-vector. p53 ubiquitination was monitored by immunoprecipitation. All cells were treated with MIG132. c: HCT116 cells were transfected with myc-RNF125, Flag-p53, and HA-ubiquitin or HA-ubiquitin (k48, k63) plasmids. At $24 \mathrm{~h}$ after transfection, MG132 was added and the cells were incubated for an additional $6 \mathrm{~h}$. Anti-Flag immunoprecipitates were analyzed using immunoblotting with HA and Flag

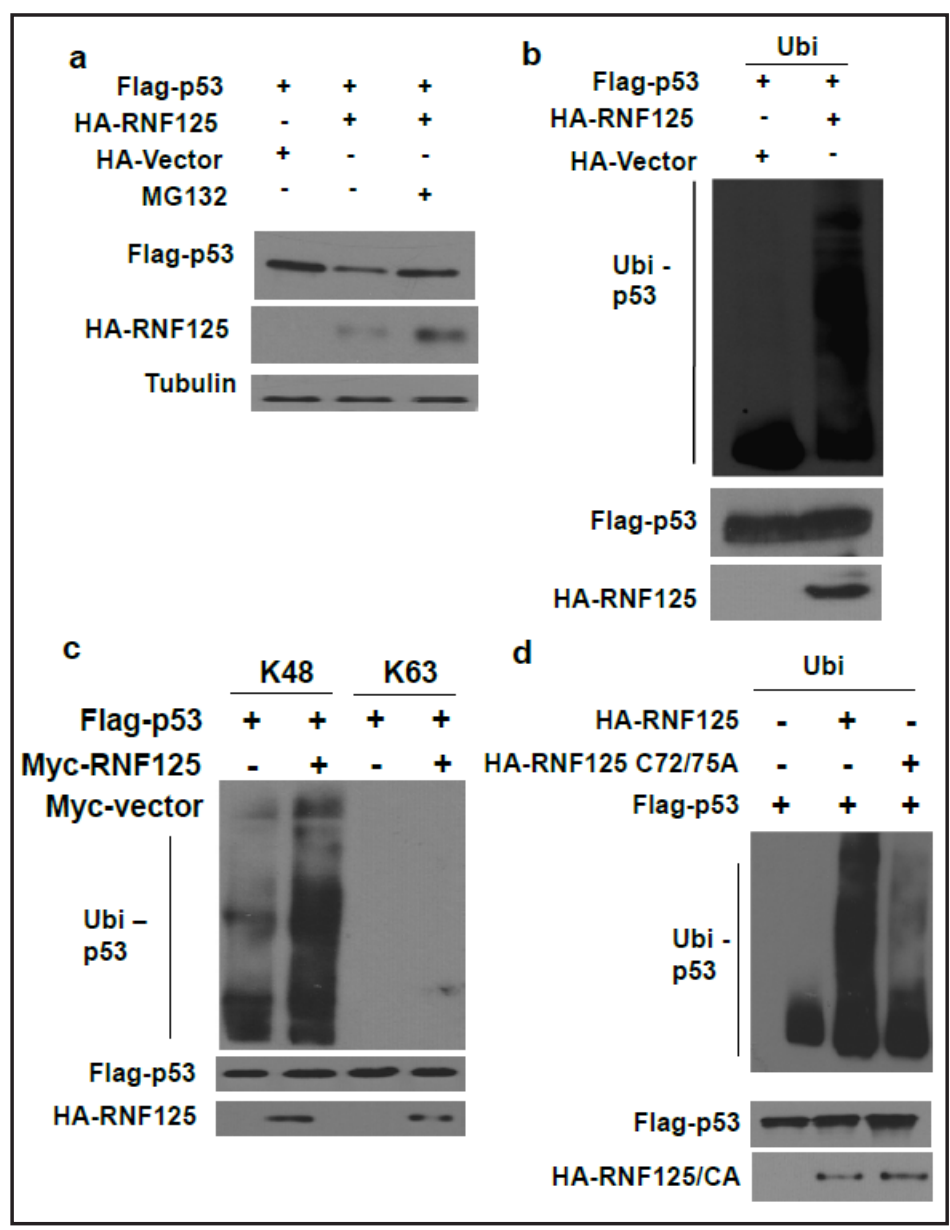

antibody. d: Ubiquitin conjugation to p53 by RNF125 and mutant RNF125. Plasmids encoding Flag-p53 and Myc-ubi were co-transfected into HCT116 cells with a plasmid encoding either WT or mutant RNF125. p53 ubiquitinatiion was monitored by immunoprecipitation. All cells were treated with MG132.

we further investigated whether RNF125 C72/75A mutant affected its interaction with p53. As shown in fig. 1e, this mutant which affects the function of RNF125 had no effect on the interaction between RNF125 and p53, suggesting the loss of function of RNF125 C72/75A mutant on p53 was not due to the loss of their interaction. Fig. $2 \mathrm{~b}$ shows the level of p53 was not changed by RNF125 C72/75A mutants overexpression, indicating the E3 ligase activity of RNF125 is necessary in downregulating expression of p53. We next examined the effect of endogenous RNF125 on p53 expression by knocking down RNF125 mRNA by using siRNA. RNF125 expression was effectively suppressed by siRNF-2 (Fig. 2c). ADR and Etop are both DNA double-strand break inducers and are commonly used as activators of p53. HCT116 cells was transfected with siRNF-2 or control siRNA and then treated with ADR $(2 \mu \mathrm{g} / \mathrm{ml})$ or Etop $(10 \mu \mathrm{M})$ for $6 \mathrm{~h}$. The results indicated that the expression of p53 was not affected in untreated cells (data not shown), however, when ADR or Etop was added, knockdown of RNF125 led to a significant increase in p53 level (Fig. 2d). As shown in Fig. 2e, RNF125 expression significantly upregulated in HCT116 cells after treatment with Etop $(10 \mu \mathrm{M})$ for $6 \mathrm{~h}$, supporting a physical role in regulating p53. These results suggested that RNF125 downregulates p53 expression.

\section{RNF125 mediates ubiquitination of p53}

Given that RNF125 levels are negatively correlated with p53 protein levels, it is possible that RNF125 induces p53 degradation through a post-translational mechanism. To test this 
Fig. 4. Suppression of p53 function by RNF125. a: HCT116 cells transiently expressing p53-responsive promoter-luciferase, HA-RNF125, GFP-RNF125 and HA-Mdm2 were analyzed for luciferase activity $36 \mathrm{~h}$ after transfection. b: HCT116 cells transiently expressing p53-responsive promoter-luciferase, HA-vector and HARNF125 wild-type or HA-RNF125 C72/75A mutant were analyzed for luciferase activity $36 \mathrm{~h}$ after transfection. c: Overexpression of RNF125 promotes HCT116 cell proliferation. HCT116 cells were transfected with HA-vector, HARNF125 or HA-RNF125 C72/75A and transfected cells were treated with Etop. Cell numbers were determined each day. d: Effects of depletion of RNF125 on HCT116 cell proliferation. HCT116 cells were transfected with HA-vector, HARNF125 or HA-RNF125 C72/75A and transfected cells were treated with Etop. Cell numbers were determined each day. HCT116 cells were treated with Etop and harvested at indicated time.

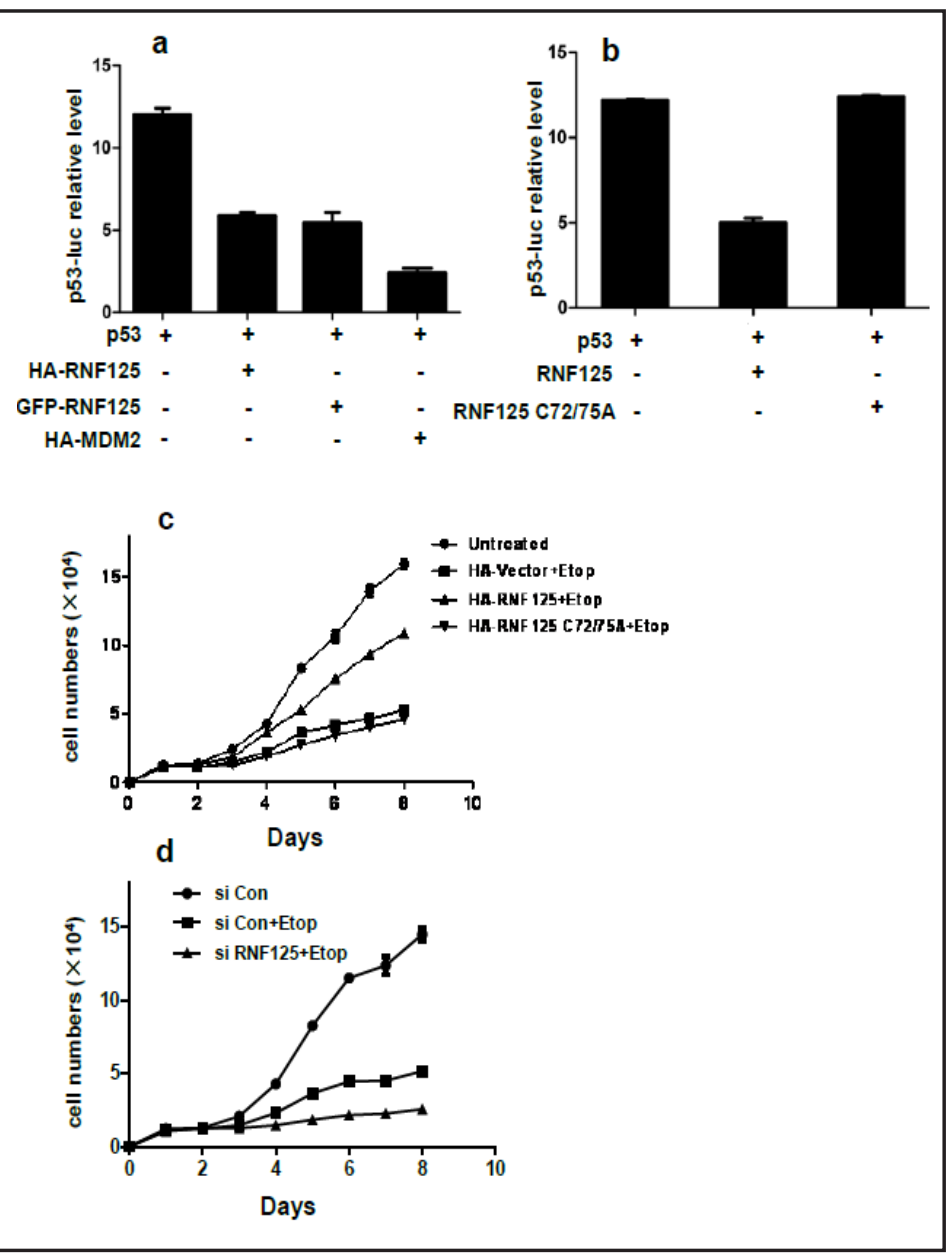

hypothesis, RNF125-mediated changes in endogenous p53 protein level were examined in HCT116 cells. Plasmids encoding a Flag-tagged version of p53 and either HA-RNF125 or HAvector were co-transfected into HCT116 cells. After culturing with or without the proteasome inhibitor MG132, expression levels of p53 and RNF125 were assessed via immunoblotting. As shown in Fig. 3a, p53 protein expression was significantly decreased in cells cultured without proteasome inhibitor, and p53 protein expression was considerably increased when protein inhibitor MG132 was added. To ascertain whether RNF125-mediated degradation of p53 by the proteasome is a direct consequence of p53 ubiquitination, HCT116 cells were transfected with plasmids encoding Flag-p53, HA-RNF125 and Myc-ubiquitin. Twenty hours after transfection, MG132 was added to a final concentration of $20 \mathrm{nM}$ for $12 \mathrm{~h}$. As shown in Fig. 3b, RNF125 led to increased ubiquitination of p53. We used Lys48 (K48-) and Lys63 (K63-) only ubiquitin expression plasmid to determine the type of ubiquitination linkage. RNF125 promoted K48-linked poly-ubiquitination of p53 but not K63-linked ubiquitination (Fig. 3c). To assess the role of E3 ligase activity of RNF125 in conjugating ubiquitin to p53, we performed a mutation analysis. Although RNF125 mediated p53 conjugation to ubiquitin (Fig. 3d, line 2), an RNF125 mutant (C72/75A) was unable to mediate p53 ubiquination (Fig. $3 \mathrm{~d}$, line 3). Hence, RNF125 ubiquitinates p53 and targets it for proteasome degradation.

RNF125 interferes with the transactivation and growth inhibitory activity of p53

To investigate the functional consequences of RNF125 interaction with p53, we first tested the effect of RNF125 on p53-mediated transcriptional activation. HCT116 cells were co-transfected with a p53-responsive luciferase reporter construct alone or in combination with HA-RNF125 vector. As shown in Fig. 4a, RNF125 significantly repressed p53-mediated 
transactivation. By transfecting MDM2 as positive control, RNF125 was found to be a weaker regulator than MDM2. RNF125 has been reported to be myristoylated at the N-terminus, leading its partial association with the membrane. To exclude possible effects by $\mathrm{N}$-terminus HA tag, we constructed RNF125-GFP plasmid in which a GFP tag is located at C-terminus. We found that p53 expression was also decreased by RNF125-GFP. Furthermore, a RNF125 mutant lost the ability to repress p53-mediated transactivation (Fig. 4b). These data indicated that RNF125 impaired the transactivation function of p53 by targeting it for degradation. Then, we examined the effect of RNF125 on growth inhibitory activity of p53. HCT116 cells were transfected with HA-vector, HA-RNF125 or HA-RNF125 C72/75A and the transfected cells were treated with $10 \mu \mathrm{M}$ Etop. Cell numbers were determined each day. When we transfected HCT116 cells with HA-RNF125, they displayed a growth advantage, whereas HCT116 cells transfected with HA-RNF125 C72/75A did not show this advantage (Fig. 4c). Furthermore, when endogenous RNF125 was knocked down by siRNA, cell growth was suppressed (Fig. 4d). These data suggest that E3 ligase activity of RNF125 is required to block the growth suppressor function of p53.

\section{Discussion}

RNF125, also known as TRAC-1 (T cell RING protein identified in activation screen), is encoded by the RNF125 gene in humans (GenBank accession no. NM_017831) and identified from a retroviral vector-based $\mathrm{T}$ cell surface activation marker screen [17]. It associates with membranes and is excluded from the nucleus through myristoylation. RNF125 bears a C3HC4 RING finger domain in the N-terminus and three zinc binding (a C2 HC and two C2H2type zinc fingers) motifs and a ubiquitin interacting motif (UIM) in the C-terminus. The UIM of RNF125 binds K48-linked poly-ubiquitin chains and is, together with the RING domain, required for auto-ubiquitination. RNF125 was recognized as belonging to a new subfamily of RING ubiquitin ligases, together with RNF114, RNF138 and RNF166 [18]. RNF125 is a RING finger E3 ubiquitin ligase with enriched expression in lymphoid tissues, specifically in $\mathrm{CD}^{+}$and $\mathrm{CD}^{+} \mathrm{T}$ cells. Previously discovered E3 ubiquitin ligases have all been shown to play negative roles in T cell activation. Recent data demonstrated RNF125 as the first E3 ubiquitin ligase that serves a positive regulatory role in T cell activation [19]. RNF125 was also found to ubiquitinate and down-modulate retinoic acid-inducible gene (RIG-I), a protein that detects viral dsDNA and induces the production of cytokines including type I interferons [16]. RNF125 was shown to be upregulated in response to interferon (IFN) and was postulated to provide a negative feedback loop for cytokine production. In addition, RNF125 has been shown to down-modulate HIV replication and to inhibit pathogeninduced cytokine production. Despite these various important roles, little is known about the RNF125 and its regulation role. In the present study, our results showed that RNF125 promotes p53 protein degradation via the ubiquitin-proteasome pathway, thereby exerting a negative regulatory role on $\mathrm{p} 53$ function.

RNF125 was identified as being overexpressed in chemoresistant epithelial ovarian cancer in differential expression experiments with high-density oligonucleotide microarrays [20]. We have now detected enhanced expression of RNF125 in HCT116 cancer cells after adding the p53-activiating chemotherapeutics Etop. In cells in which RNF125 expression had been knocked down, the addition of chemotherapeutics significantly reduced cancer cell growth, and adding wild-type RNF125 restored cancer cell growth. In cells bearing a RNF125 mutant lacking E3 ligase activity, cancer cell growth was not restored by adding wild-type RNF125 (data not shown). It can be observed that RNF125 possibly participats in cancer tumorigenesis, development and chemoresistance, but its mechanisms remain to be elucidated.

p53 is a central hub in a molecular network controlling cell proliferation and death in response to potentially oncogenic conditions, and a wide array of covalent modifications and protein interactions modulate the nuclear and cytoplasmic activities of p53. One of the

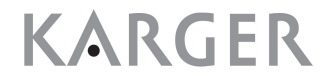


key mechanisms is regulation through control of protein stability. Under normal growth conditions, p53 protein levels are kept low as a consequence of rapid degradation via the proteasome. Mdm2 is the major E3 ubiquitin ligase for $\mathrm{p} 53$, regulating its stability by directly assembling polyubiquitin chains on p53 and so targeting it for proteasomal degradation [21]. In addition to $\mathrm{Mdm} 2$, a number of other ubiquitin ligases have been shown to regulate the stability of p53, including COP1, Pirh2, ARFBP1, CHIP, Synoliolin, CARP1, CARP2 and TRIM24 [21]. However, none of these proteins can compensate for the loss of Mdm2 function in vivo. It is generally considered that Mdm2, COP1, Pirh2, ARF-BP1, and other ubiquitin ligases constitute an array of E3 ligases upon which the cell can call to regulate and maintain p53 levels. These suggest that both Mdm2-dependent and Mdm2-independent mechanisms are used cooperatively by cells to tightly regulate p53. In the present study, we demonstrated that RNF125 physically interacts with p53 and downregulates its expression. Further studies are needed to clarify the context under which these E3 ligasess control p53 and the role of RNF125 also remains to be elucidated.

In summary, we have shown that RNF125 directly interacts with p53 and regulates its function. How this negative regulator hierarchically incorporates the regulatory function toward efficacy of $\mathrm{p} 53$ proteins still remains to be elucidated. This study raises the possibility that overexpression of RNF125 in chemoresistant cancer cells may provide yet another mechanism to inactivate wild-type p53.

\section{Disclosure Statement}

None.

\section{References}

Levine AJ, Oren M: The first 30 years of p53: Growing ever more complex. Nat Rev Cancer 2009;9:749-758. Brady CA, Attardi LD: P53 at a glance. J Cell Sci 2010;123:2527-2532.

Hock AK, Vousden KH: The role of ubiquitin modification in the regulation of p53. Biochim Biophys Acta 2014;1843:137-149.

Haupt Y, Maya R, Kazaz A, Oren M: Mdm2 promotes the rapid degradation of p53. Nature 1997;387:296299.

Honda R, Yasuda H: Association of p19(arf) with mdm2 inhibits ubiquitin ligase activity of mdm2 for tumor suppressor p53. EMBO J 1999;18:22-27.

Kubbutat MH, Jones SN, Vousden KH: Regulation of p53 stability by mdm2. Nature 1997;387:299-303. Clegg HV, Itahana K, Zhang Y: Unlocking the mdm2-p53 loop: Ubiquitin is the key. Cell Cycle 2008;7:287292.

Fang S, Jensen JP, Ludwig RL, Vousden KH, Weissman AM: Mdm2 is a ring finger-dependent ubiquitin protein ligase for itself and p53. J Biol Chem 2000;275:8945-8951.

Momand J, Zambetti GP, Olson DC, George D, Levine AJ: The mdm-2 oncogene product forms a complex with the p53 protein and inhibits p53-mediated transactivation. Cell 1992;69:1237-1245.

Lang GA, Iwakuma T, Suh YA, Liu G, Rao VA, Parant JM, Valentin-Vega YA, Terzian T, Caldwell LC, Strong LC, El-Naggar AK, Lozano G: Gain of function of a p53 hot spot mutation in a mouse model of li-fraumeni syndrome. Cell 2004;119:861-872.

1 Olive KP, Tuveson DA, Ruhe ZC, Yin B, Willis NA, Bronson RT, Crowley D, Jacks T: Mutant p53 gain of function in two mouse models of li-fraumeni syndrome. Cell 2004;119:847-860.

Leng RP, Lin Y, Ma W, Wu H, Lemmers B, Chung S, Parant JM, Lozano G, Hakem R, Benchimol S: Pirh2, a p53-induced ubiquitin-protein ligase, promotes p53 degradation. Cell 2003;112:779-791. 
13 Dornan D, Shimizu H, Mah A, Dudhela T, Eby M, O'Rourke K, Seshagiri S, Dixit VM: Atm engages autodegradation of the e3 ubiquitin ligase cop1 after DNA damage. Science 2006;313:1122-1126.

14 Chen D, Kon N, Li M, Zhang W, Qin J, Gu W: Arf-bp1/mule is a critical mediator of the arf tumor suppressor. Cell 2005;121:1071-1083.

15 Yang W, Rozan LM, McDonald ER, 3rd, Navaraj A, Liu JJ, Matthew EM, Wang W, Dicker DT, El-Deiry WS: Carps are ubiquitin ligases that promote mdm2-independent p53 and phospho-p53ser20 degradation. J Biol Chem 2007;282:3273-3281.

16 Arimoto K, Takahashi H, Hishiki T, Konishi H, Fujita T, Shimotohno K: Negative regulation of the rig-i signaling by the ubiquitin ligase rnf125. Proc Natl Acad Sci U S A 2007;104:7500-7505.

-17 Chu P, Pardo J, Zhao H, Li CC, Pali E, Shen MM, Qu K, Yu SX, Huang BC, Yu P, Masuda ES, Molineaux SM, Kolbinger F, Aversa G, de Vries J, Payan DG, Liao XC: Systematic identification of regulatory proteins critical for t-cell activation. J Biol 2003;2:21.

18 Giannini AL, Gao Y, Bijlmakers MJ: T-cell regulator rnf125/trac-1 belongs to a novel family of ubiquitin ligases with zinc fingers and a ubiquitin-binding domain. Biochem J 2008;410:101-111.

19 Zhao H, Li CC, Pardo J, Chu PC, Liao CX, Huang J, Dong JG, Zhou X, Huang Q Huang B, Bennett MK, Molineaux SM, Lu H, Daniel-Issakani S, Payan DG, Masuda ES: A novel e3 ubiquitin ligase trac-1 positively regulates $\mathrm{t}$ cell activation. J Immunol 2005;174:5288-5297.

20 Ju W, Yoo BC, Kim IJ, Kim JW, Kim SC, Lee HP: Identification of genes with differential expression in chemoresistant epithelial ovarian cancer using high-density oligonucleotide microarrays. Oncol Res 2009;18:47-56.

21 Lee JT, Gu W: The multiple levels of regulation by p53 ubiquitination. Cell Death Differ 2010;17:86-92. 